\title{
Potencial evocado auditivo para diagnóstico de surdez bilateral em dois cães
}

\author{
Auditory evoked potential to diagnose of bilateral deafness in two dogs \\ Mariana Isa Poci Palumbo ${ }^{I}$ Luiz Antonio de Lima Resende ${ }^{\text {II }}$ Luiz Henrique de Araújo Machado ${ }^{\text {III }}$ \\ Alexandre Secorun Borges ${ }^{\mathrm{II}}{ }^{*}$
}

\section{- NOTA-}

\section{RESUMO}

O potencial auditivo evocado de tronco encefálico é um método eletrodiagnóstico não invasivo que permite avaliação objetiva do estado auditivo, da orelha média ao tronco encefálico, captando a atividade elétrica do sistema auditivo, gerada a partir de um estímulo sonoro específico. $O$ uso desse teste não é difundido em animais no Brasil. Sendo assim, o objetivo do presente trabalho é relatar o diagnóstico de surdez bilateral em dois cães sem raça definida, com a utilização do potencial evocado auditivo de tronco encefálico.

Palavras-chave: surdez, potencial evocado, sistema auditivo, cães.

\section{ABSTRACT}

The brainstem auditory evoked potential is a noninvasive electrodiagnostic test allowing an objective assessment of the hearing status, by capturing the electrical activity of the auditory system, from the middle ear to the brainstem, generated after a specific sound stimulus is performed. The use of this test is not common in animals in Brazil. Therefore, the objective of this study is to report the diagnosis of bilateral deafness in two mongrel dogs, using the brainstem auditory evoked potential.

Key words: deafness, evoked potential, auditory system, dogs.
A surdez é um problema subestimado na clínica de pequenos animais (FÉRNANDEZ \& BERNARDINI, 2010). Existem duas categorias de surdez: condução e neurossensorial. A surdez por condução ocorre quando há falha na transmissão adequada da vibração do som para dentro da orelha interna (STRAIN, 1999). Os exemplos incluem oclusão do canal auricular externo por debris ceruminosos, destruição da membrana timpânica e otite externa/média grave, dentre outros fatores (ROSYCHUK \& LUTTGEN, 2004). A surdez neurossensorial resulta de anormalidades das estruturas do ouvido interno, do nervo vestibulococlear e/ou das vias anatômicas do tronco encefálico, incluindo tálamo e córtex (SIMS, 1990). As principais causas de surdez neurossensorial incluem: surdez hereditária, lesão neuronal por substâncias ototóxicas e surdez senil ou relacionada à idade (ROSYCHUK \& LUTTGEN, 2004).

A avaliação da audição em cães pode ser realizada observando-se as respostas comportamentais a estímulos sonoros (LUTTGEN, 1994). Nesse caso, o teste é considerado positivo se o animal virar a cabeça em resposta a um estímulo auditivo fora de seu campo visual (COX, 2002). Entretanto, esses testes são inviáveis para filhotes, cães agitados, desatentos e com alterações de consciência, além de não permitirem o

'Faculdade de Medicina Veterinária e Zootecnia (FMVZ), Universidade Estadual Paulista “Júlio de Mesquita Filho” (UNESP), Botucatu, SP, Brasil.

IIDepartamento de Neurologia, Psicologia e Psiquiatria, Faculdade de Medicina de Botucatu (FMB), UNESP, Botucatu, SP, Brasil.

IIIFMVZ, UNESP, 18618-970, Botucatu, SP, Brasil. E-mail: asborges@fmvz.unesp.br *Autor para correspondência. 
diagnóstico de surdez unilateral (STRAIN, 1996; STRAIN, 1999).

O potencial evocado auditivo de tronco encefálico é um teste eletrofisiológico que capta e registra as atividades elétricas do sistema auditivo, desde a cóclea até o tronco encefálico, geradas a partir de um estímulo sonoro. Os potenciais elétricos são registrados com quatro a seis ondas identificadas por algarismos romanos (I, II, III, IV, V e VI) que aparecem nos 10 primeiros milissegundos após o estímulo (WEBB, 2009). Na Europa e na América do Norte, a resposta auditiva evocada de tronco encefálico é comumente empregada para o diagnóstico de surdez por ser um método objetivo, não-invasivo e seguro (WILSON \& MILLS, 2005), porém seu uso ainda é pouco difundido no Brasil. Sendo assim, o objetivo do presente trabalho é relatar o diagnóstico de surdez em dois cães sem raça definida, com a utilização do potencial evocado auditivo de tronco encefálico.

Foram atendidos pelo Setor de Neurologia Veterinária do Hospital Veterinário da Universidade Estadual Paulista “Júlio de Mesquita Filho”, campus de Botucatu, dois cães sem raça definida com suspeita clínica de surdez bilateral congênita. Em ambos os casos, os proprietários assinaram o termo de consentimento livre e esclarecido. O primeiro animal era um cão macho de 6 meses de idade com olhos castanhos e pelame branco. O segundo caso era uma fêmea de 6 anos de idade com olhos e pelame castanhos. Em ambos os casos, os proprietários e tratadores suspeitavam de surdez bilateral, porque, desde quando eram filhotes, não atendiam a chamados e não percebiam sons evidentes, como o toque da campainha ou barulho de fogos de artifício.

Durante a anamnese, constatou-se que medicações ototóxicas nunca haviam sido utilizadas e não havia histórico compatível com otite (prurido otológico ou meneios cefálicos). Os animais foram submetidos aos exames neurológico, segundo MARCONDES (2008), e otoscópico, segundo COLE (2004), e não foram observadas alterações. Foram realizados alguns testes ambulatoriais para avaliar a acuidade auditiva, como bater palmas e chamar os cães fora do campo de visão, e ambos não responderam aos estímulos.

Os potenciais evocados foram realizados nos dois casos, no Laboratório de Eletroneurodiagnóstico do Departamento de Clínica Veterinária, FMVZ- UnespBotucatu com a utilização de equipamento marca Viasys Healthcare $^{\circledR}$, modelo Teca Synergy de dois canais. Em um dos animais, a realização do exame foi possível mediante contenção física, o outro necessitou de sedação com midazolan intramuscular $\left(0,2 \mathrm{mg} \mathrm{kg}^{-1} \mathrm{PV}\right)$ e metadona $\left(0,2 \mathrm{mg} \mathrm{kg}^{-1} \mathrm{PV}\right)$ devido à agitação. Os animais foram posicionados em decúbito esternal. Os eletrodos de registro foram colocados na região rostral ao tragus da orelha a ser testada (G1) e o de referência (G2) foi posicionado no vértice do crânio (Cz) (KAY et al., 1984). O fio terra foi colocado na região cervical dorsal (EGER \& LINDSAY, 1997). Foram utilizados filtros com banda passante de $200 \mathrm{~Hz}$ a $3.000 \mathrm{~Hz}$, sensibilidade de $2.5 \mathrm{uV} \mathrm{cm} \mathrm{cm}^{-1}$ e varredura de $1 \mathrm{~ms} \mathrm{~cm}^{-1}$. Fones de ouvido externos foram posicionados sobre as orelhas do animal. Utilizaram-se “cliques” de rarefação de $0.2 \mathrm{~ms}$, de $85 \mathrm{~dB}$, na frequência de $13 \mathrm{~Hz}$, com mascaramento de ruído branco contralateral de 40dB. Em cada lado, foram obtidas pelo menos duas séries de 500 promediações. Em ambos os casos, o exame não evidenciou ondas I, III e V (com linhas de base de boa qualidade técnica, após duas séries de 500 promediações). A figura 1 mostra o potencial evocado auditivo normal desta espécie e traçados obtidos nos animais desse relato.

A maioria dos casos de surdez neurossensorial completa deve-se à doença da cóclea e/ou do nervo periférico. Como resultado, nenhuma onda é gerada na obtenção dos potenciais, como observado nos exames dos animais desse relato (STRAIN, 1996; ROSYCHUK \& LUTTGEN, 2004). A ausência de atividade elétrica nas vias auditivas observada nesses casos já foi anteriormente relatada em casos de surdez congênita em cães (KANG et al., 2008).

A maioria dos animais não requer contenção química para a realização do exame, entretanto, o uso de medicação sedativa faz com que o ruído de fundo (da atividade cortical) interfira menos, evitando-se artefatos e conseguindo-se respostas mais limpas, em que as latências das diferentes ondas são identificadas mais facilmente (LASMAR et al., 1994). Segundo STRAIN (1996), a resposta não é afetada pela sedação, que foi necessária em um dos animais desse relato, que era muito agitado e não permitiu a realização do exame apenas com a contenção física.

Normalmente, quando há perda parcial da audição (em casos de uso de medicamentos ototóxicos ou surdez senil), há um aumento da latência da onda I, e a amplitude das ondas é diminuída (STRAIN, 1996), porém, isso não foi observado nos casos relatados, que foram mais compatíveis com surdez neurossensorial completa. A idade de surgimento dos sinais clínicos, a ausência de alterações no exame otoscópico e a exclusão do uso de medicamentos neurotóxicos sugeriram o diagnóstico de surdez congênita.

A surdez neurossensorial congênita é uma doença hereditária comumente observada em cães com pigmentação branca (MUHLE et al., 2002) e olhos de coloração clara (FÉRNANDEZ \& BERNARDINI, 2010). Sua ocorrência foi documentada em pelo menos 80 raças (STRAIN, 2004). Em 2000, COPPENS e colaboradores 


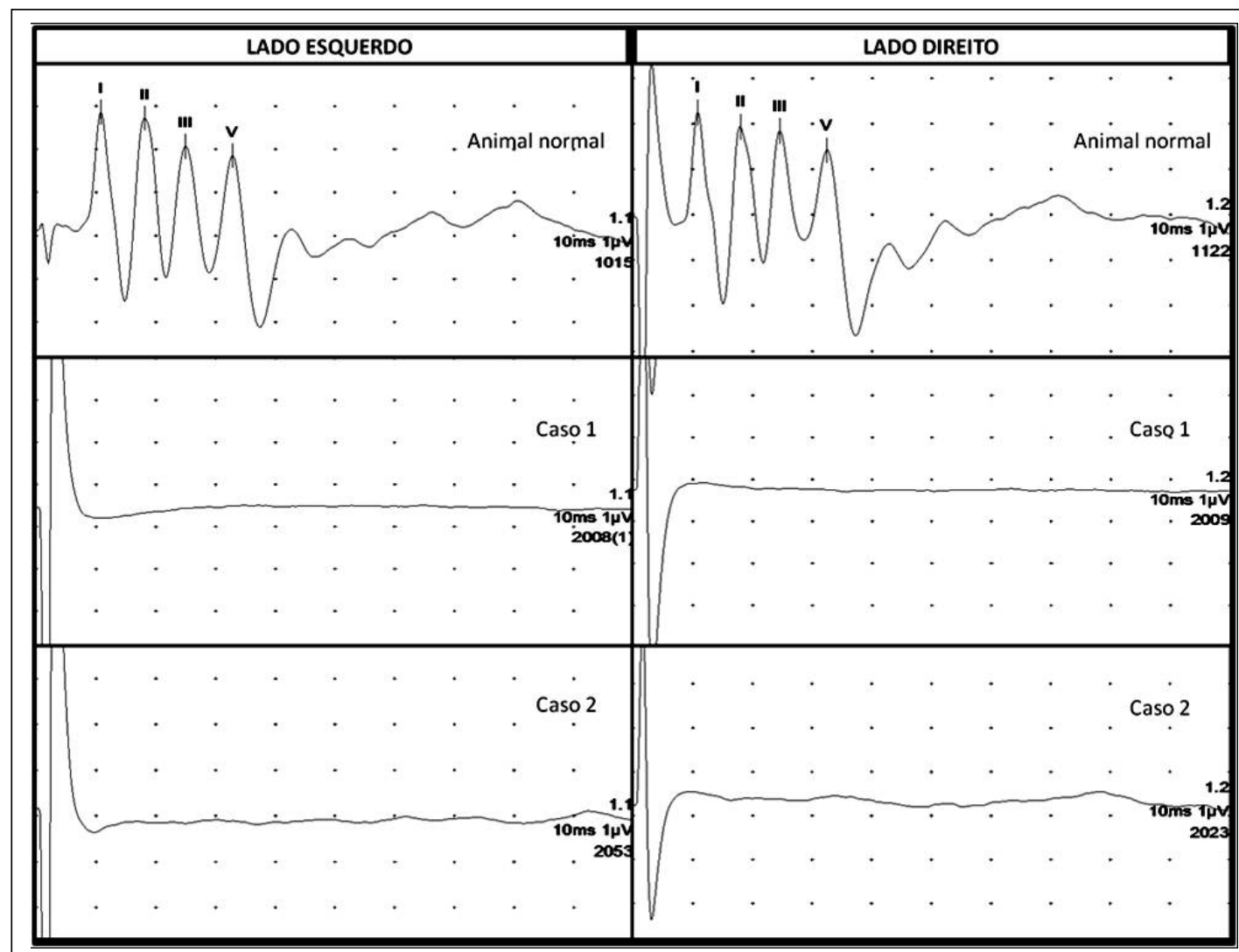

Figura 1 - Potencial auditivo evocado realizado em um cão normal e os traçados obtidos nos animais desse relato (Casos 1 e 2). Note que as ondas observadas no exame do animal normal (ondas I, II, III e V) estão ausentes no traçado dos animais com provável surdez.

relataram a ocorrência de surdez em dois cães da raça Maltês Terrier e, segundo WOOD \& LAKHANI (1998), cerca de $20 \%$ dos animais da raça Dálmata apresentam surdez unilateral e $10 \%$ têm surdez bilateral. Acreditase que este trabalho representa pequena contribuição a esta área específica da Medicina Veterinária, porque não se encontra descrições de surdez bilateral em cães sem raça definida, comuns na medicina veterinária do Brasil.

\section{COMITÊ DE ÉTICA E BIOSSEGURANÇA}

O número do protocolo do comitê de ética é: 117/ 2009.

\section{AGRADECIMENTO}

Agradecemos o apoio fornecido pela Fundação de Amparo à Pesquisa do Estado de São Paulo (FAPESP) na forma de bolsa (Processo 2009/12290-8) e auxílio (Processo 2010/ 00518-1).

\section{REFERÊNCIAS}

COLE, L.K. Otoscopic evaluation of the ear canal. Veterinary Clinics Small Animal Prcatice, v.34, p.397-410, 2004. Disponível em: <http://www.ncbi.nlm.nih.gov/pubmed/ 15062615>. Acesso em: 18 jun. 2011. doi: 10.1016/ j.cvsm.2003.10.004.

COX, C. Investigation of hearing loss in dogs. In Practice, v.1 p.494-501, 2002. Disponível em: <http:// inpractice.bmj.com/content/24/9/494>. Acesso em: 18 jun. 2011. doi: 10.1136/inpract.24.9.494.

COPPENS, A.G. et al. Bilateral deafness in a Maltese Terrier and a Great Pyrenean Puppy: inner ear morphology. Journal of Comparative Pathology, v.122, p.223-228, 2000. Disponível em: <http://www.ncbi.nlm.nih.gov/pubmed/ 10684693>. Acesso em: 18 jun. 2011. doi: 10.1053/ jсра.2002.0596.

EGER, C.E.; LINDSAY, P. Effects of otitis on hearing in dogs characterized by brainstem auditory evoked response testing. Journal of Small Animal Practice, v.38, p.380-386, 1997. 
Disponível em: <http://www.ncbi.nlm.nih.gov/pubmed/ 9322176>. Acesso em: 18 jun. 2011.

FERNÁNDEZ, V.L.; BERNARDINI, M. Síndromes vestibulares e alterações da audição. In: Neurologia em cães gatos. São Paulo: Medvet, 2010. p.391-414.

KANG, B. et al. Bilateral congenital deafness in a bull terrier; brainstem auditory evoked response findings. Journal of Veterinary Clinics, v.25, n.6, p.506-509, 2008. Disponível em: <http://210.101.116.28/W_kiss6/33301496_pv.pdf >. Acesso em: 18 jun. 2011.

KAY, R. et al. Hearing in the dog as assessed by auditory brainstem evoked potentials. Veterinary Records, v.114, p.81-84, 1984. Disponível em: <http:// veterinaryrecord.bmj.com/content/114/4/81>. Acesso em: 18 jun. 2011.

LASMAR, A. et al. Topodiagnóstico das disacusias sensoriais: audiometria supraliminar. In: FILHO, O.L.; CAMPOS, C.A. Tratado de otorrinolaringologia. São Paulo: Roca, 1994. p.560-598.

LUTTGEN, P.J. Deafness in the dog and cat. Veterinary Clinics of North America, v.24, n.5, p.981-989, 1994 Disponível em: <http://www.ncbi.nlm.nih.gov/pubmed/ 7817497>. Acesso em: 18 jun. 2011.

MARCONDES, M. Semiologia do sistema nervoso de pequenos animais. In: FEITOSA, F.L.F. Semiologia veterinária: a arte do diagnóstico. São Paulo: Roca, 2008. p.411-460.

MUHLE, A.C. et al. Further contributions to the genetic aspect of congenital sensorineural deafness in Dalmatians. Veterinary Journal, v.163, p.311-318, 2002. Disponível em: <http:// w w w. sciencedirect.com/science/article / pi i / S1090023301906616>. Acesso em: 18 jun. 2011. doi: 10.1053/ tvjl.2001.0661.

SIMS, M.H. Evoked response audiometry in dogs. Proceedings in Veterinary Neurology, v.1, n.1, p.275-283, 1990.
Disponível em: <http://www.cabdirect.org/abstracts/ 19902214809.html?freeview=true>. Acesso em: 18 jun. 2011.

STRAIN, G.M. Aetiology, prevalence and diagnosis of deafness in dogs and cats. British Veterinary Journal, v.152, n.1, p.17-36, 1996. Disponível em: <http://www.sciencedirect.com/ science/article/pii/S0007193596800832.> Acesso em: 18 jun. 2011. doi: 10.1016/S0007-1935(96)80083-2.

STRAIN, G.M. Deafness prevalence and pigmentation and gender associations in dog breeds at risk. Veterinary Journal, v.167, p.23-32, 2004. Disponível em: <http:// www.ncbi.nlm.nih.gov/pubmed/14623147>. Acesso em: 18 jun. 2011. doi: 10.1016/S1090-0233(03)00104-7.

STRAIN, G.M. Congenital deafness and its recognition. Veterinary Clinics of North America: Small Animal Practice, v.29, p.895-907, 1999. Disponível em: <http:// www.ncbi.nlm.nih.gov/pubmed/10390790>. Acesso em: 18 jun. 2011.

ROSYCHUK, R.A.W.; LUTTGEN, P. Doenças dos ouvidos. In: ETTINGER, S.J.; FELDMAN, E.C. Tratado de medicina interna veterinária: doenças do cão e do gato. 5.ed. Rio de Janeiro: Guanabara Koogan, 2004. p.1042-1059.

WEBB, A.A. Brainstem auditory evoked response (BAER) testing in animals. Canadian Veterinary Journal, v.50, p.313-318, 2009. Disponível em: <http:// www.ncbi.nlm.nih.gov/pmc/articles/PMC2643461>. Acesso em: 18 jun. 2011.

WILSON, W.J.; MILLS, P.C. Brainstem auditory-evoked response in dogs. American Journal of Veterinary Research, v.66, n.12, p.2177-2187, 2005.

WOOD, J.L.N.; LAKHANI, K.H. Deafness in Dalmatians: does sex matter? Preventive Veterinary Medicine, v.36, p.39-50, 1998. Disponível em: <http://www.sciencedirect.com/ science/article/pii/S0167587798000749>. Acesso em: 18 jun. 2011. doi:10.1016/S0167-5877(98)00074-9. 